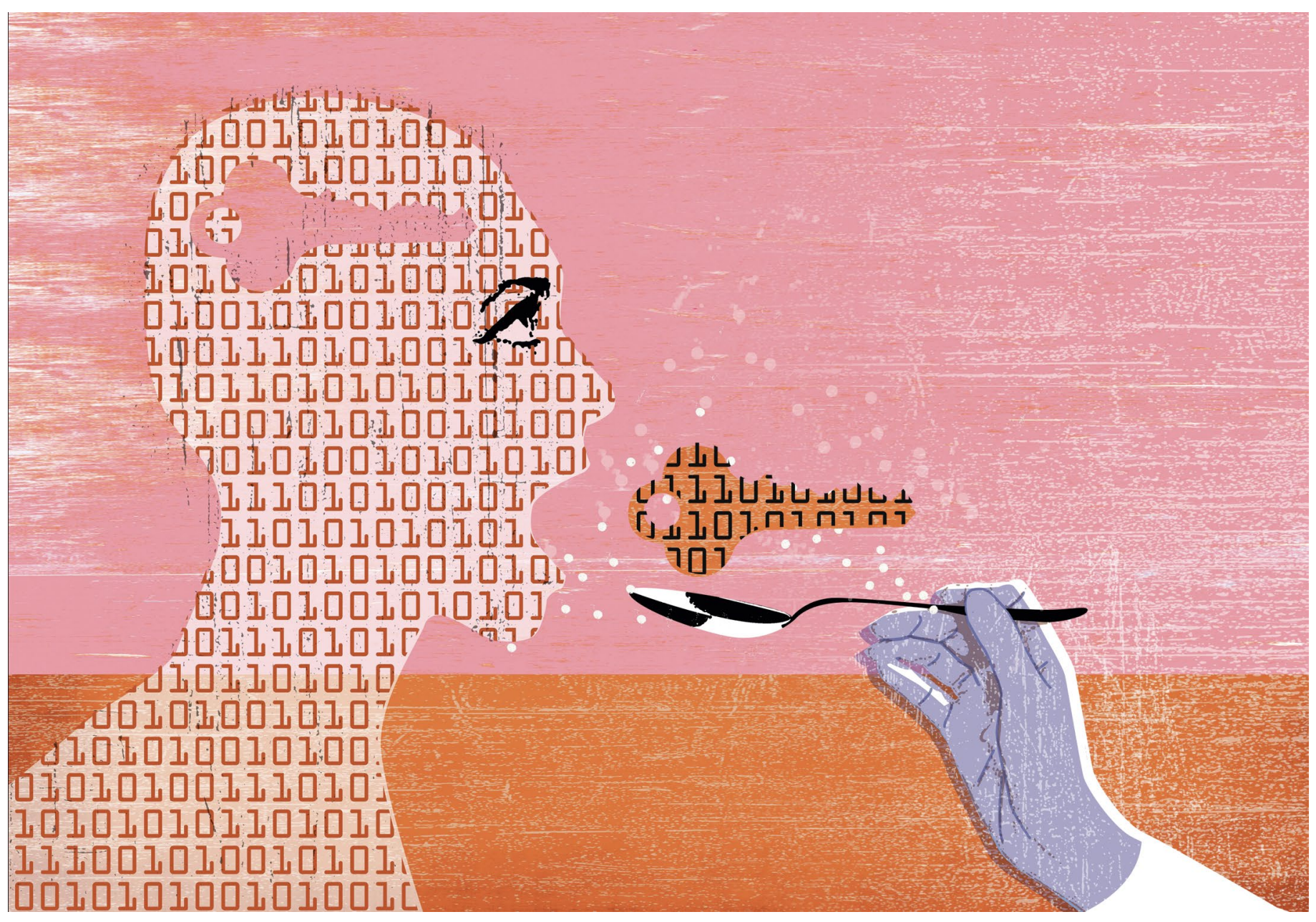

\title{
Looking forward 25 years: the future of medicine
}

To celebrate the end of our 25th anniversary year, we asked thought leaders and experts in the field to answer one question: What will shape the next 25 years of medical research?

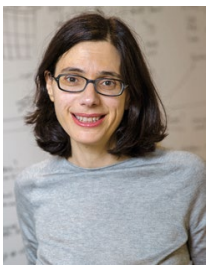

Credit: Casey Atkins

\section{Aviv Regev}

Core member and chair of the faculty, Broad Institute of MIT and Harvard; director, Klarman Cell Observatory, Broad Institute of MIT and Harvard; professor of biology, MIT; investigator, Howard Hughes Medical Institute; founding co-chair, Human Cell Atlas.

For many years, biology and disease appeared 'too big' to tackle on a broad level: with millions of genome variants, tens of thousands of disease-associated genes, thousands of cell types and an almost unimaginable number of ways they can combine, we had to approximate a best starting point-choose one target, guess the cell, simplify the experiment.

But we are now on the cusp of an inflection point, where the 'bigness' of biomedicine turns into an advantage. We are beginning to see advances towards these goals already, in polygenic risk scores, in understanding the cell and modules of action of genes through genome-wide association studies (GWAS), and in predicting the impact of combinations of interventions. Going forward, our success in harnessing bigness will rely on our ability to leverage structure, prediction and expanded data scale. Disease is highly structured at the molecular, genetic, gene program, cell and tissue levels; acknowledging and understanding this structure can help us reduce the overwhelming lists of genes and variants to a manageable number of meaningful gene modules. We cannot test every possible combination, so we need algorithms to make better computational predictions of experiments we have never performed in the lab or in clinical trials. But only when data are truly big, scaled massively and rich in content, will we have the most effective structuring and prediction 
power towards building a much-needed Roadmap of Disease for patients.

To achieve this, we need to invest in building the right initiatives-like the Human Cell Atlas and the International Common Disease Alliance-and in new experimental platforms: data platforms and algorithms. But we also need a broader ecosystem of partnerships in medicine that engages interaction between clinical experts and mathematicians, computer scientists and engineers who together will bring new approaches to drive experiments and algorithms to build this Roadmap.

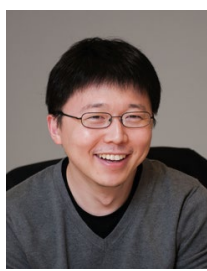

\section{Feng Zhang}

PhD investigator, Howard Hughes Medical Institute; core member, Broad Institute of MIT and Harvard; James and Patricia Poitras Professor of Neuroscience, McGovern Credit: Stan Grazier, Broad Institute for Brain Research, MIT.

Although it is difficult to pinpoint an exact value, it is safe to estimate that more than 250 patients have been treated with gene therapies for monogenic diseases for which there previously were no treatment options. Add in the patients who have received CAR-T therapy, and that number rises into the thousands. This is an enormous success, and it represents the beginning of a fundamental shift in medicine away from treating symptoms of disease and toward treating disease at its genetic roots.

Gene therapy has been under development for more than 30 years, but several recent major advances have tipped the scales toward clinical feasibility, including improved delivery methods and the development of robust molecular technologies for gene editing in human cells. In parallel, affordable genome sequencing has accelerated our ability to identify the genetic causes of disease. With these advances, the stage is set for the widespread use of gene therapy. Already, nearly 1,000 clinical trials testing gene therapies are ongoing, and the pace of clinical development is likely to accelerate.

To fulfil the potential of gene therapy and ensure that all patients have access to this revolutionary treatment, we will need to continue developing delivery approaches that are practical and widely usable, to refine molecular technologies for gene editing, to push our understanding of gene function in health and disease forward, and to engage with all members of society to openly discuss the risks and benefits of gene therapy.

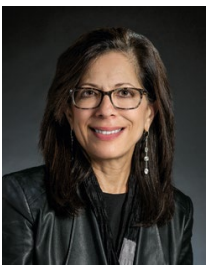

\section{Elizabeth Jaffee}

Dana and Albert "Cubby"

Broccoli Professor of

Oncology, Johns Hopkins

School of Medicine; deputy

director, Sidney Kimmel

Comprehensive Cancer

Center at Johns Hopkins.

Credit: Fred

Dubs, Johns

Hopkins

University

"An ounce of prevention is worth a pound of cure." Benjamin Franklin said this in reference to fire safety, but it can easily be applied

to health too. The twentieth century saw amazing advances aimed at preventing the onset of disease-including vaccines and risk-factor interventions-nearly doubling life expectancy worldwide. Only two decades into the twenty-first century, healthcare has already entered its next phase of rapid advancements. By using precision medicine technologies, genetic vulnerabilities to chronic and deadly diseases at the individual level can now be identified, potentially preempting disease decades later.

My hope for the next 25 years is that someday a single blood test could inform individuals of the diseases they are at risk of (diabetes, cancer, heart disease, etc.) and that safe interventions will be available. I am particularly excited about the possibility of developing cancer vaccines. Vaccines targeting the causative agents of cervical and hepatocellular cancers have already proven to be effective. With these technologies and the wealth of data that will become available as precision medicine becomes more routine, new discoveries identifying the earliest genetic and inflammatory changes occurring within a cell as it transitions into a pre-cancer can be expected. With these discoveries, the opportunities to develop vaccine approaches preventing cancers development will grow.

But, as is the case today, prevention technologies can only be fully successful if they are widely available, to reduce unnecessary morbidity and mortality and healthcare costs and further raise life expectancy. Global accessibility is key to reduce global disparities. For these strategies to work, funding agencies should consider prioritizing prevention strategies.

\section{Jeremy Farrar}

Director, Wellcome Trust.

Politics, demographics, economics, climate-how the world changes and interacts fundamentally affects all of us. Research is part of that and can help provide

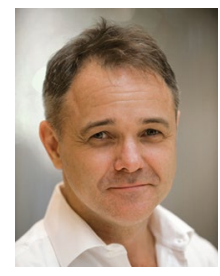

Credit: Wellcome Trust solutions to the great challenges we face, but only if the three pillars of science, innovation and society come together in an environment where people and teams can thrive. We must therefore take the opportunity today to shape how the culture of research will develop over the next 25 years.

Building a career in research can be incredibly rewarding, yet it often comes at a cost. The drive for research excellence-to which Wellcome has certainly contributedhas created a culture that cares more about what is achieved than how it is achieved. We can do better, and building a creative, inclusive and open research culture will unleash greater discoveries with greater impact.

Changing culture requires us to acknowledge the issue and then make a long-term commitment. As an independent foundation, Wellcome is able to acknowledge the issue and make that commitment. This is a permanent shift in our thinking. Working openly with, and as part of, the wider research community, we aim to make research inclusive, more inspiring, more fun, more rewarding. As a result, it will contribute even more to making the world a healthier place to live.

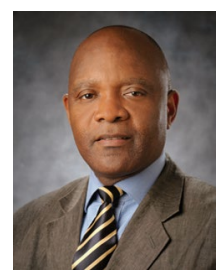

John Nkengasong Director, Africa Centres for Disease Control and Prevention.

Population wise, Africa is the continent of the future. By 2050, it is estimated that its

Credit: John Nkengasong population will be 2.5 billion people. This means that one in every four persons in the world might be an African, with rapidly growing economies and a rising middle class. These demographic changes have important implications for both communicable and noncommunicable disease patterns, including emerging and re-emerging infectious diseases; resistance to antibiotics; and rising rates of cancers, diabetes, cardiovascular diseases and maternal and child deaths. To meet its health challenges by 2050 , the continent will have to be innovative in order to leapfrog toward solutions in public health.

Precision medicine will need to take center stage in a new public health orderwhereby a more precise and targeted approach to screening, diagnosis, treatment 
and, potentially, cure is based on each patient's unique genetic and biologic make-up. For example, universal newborn screening and a more accurate analysis of causes of death in this age group could be established to curb under-five mortality; genetic screening programs could help avoid progression towards aggressive cancers; and medicine side effects could be reduced if tests could predict negative reactions and enable caregivers to proactively prescribe alternative treatments.

In Africa, precision medicine should not be seen from the lens of sequencing whole genomes, diagnosing DNA abnormalities and developing medications targeted to very small populations. Rather, African countries should begin pursuing policy approaches and partnerships to advance precision medicine to meet the African Union's Agenda 2063 goals. This includes the integration of precision medicine approaches into national strategies to improve healthcare-including genomic data policy - and increase diagnostic capacity, and the creation of biobanks, such as H3Africa, that encompass both physical and bioinformatics facilities.

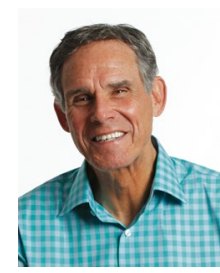

Credit: Scripps

Research

Institute

\section{Eric Topol}

Executive vice-president, Scripps Research Institute; founder and director, Scripps Research Translational Institute.

Twenty-five years ago, the World Wide Web was just getting off the ground. Therefore, when thinking of the medical research landscape in 25 years, it is reasonable to think big and without limits.

In 2045, I hope we will have developed a planetary health infrastructure based on deep, longitudinal, multimodal human data, ideally collected from and accessible to as many as possible of the $9+$ billion people projected to then inhabit the Earth.

This infrastructure, by using hybrid artificial intelligence (AI) modelsincluding various deep neural networks, federated AI, nearest-neighbor analysis and systems yet to be developed-could provide individualized guidance for the prevention and optimal management of medical conditions, acting as a virtual medical coach for patients and a platform for clinicians to review a patient's real-time, real-world, extensive and cumulative dataset.

Some have projected that, by this juncture, artificial general intelligence (AGI) will have been developed, giving machines

enhanced capabilities to perform functions that are not feasible now. Notwithstanding that uncertainty, it is likely that machines' ability to ingest and process biomedical text at scale-such as the corpus of the up-to-date medical literature-will be used routinely by physicians and patients. Accordingly, the concept of a learning health system will be redefined.

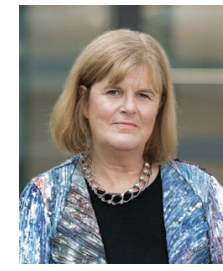

Credit: Max

Planck Institute for Biology of Ageing

especially in women.

\section{Linda Partridge}

Professor, Max Planck Institute for Biology of Ageing.

Human life expectancy has increased over the past 170 years in many parts of the world. Unfortunately, the healthy lifespan has not, and the period of life when a person lives with disability and illness at the end of life is growing,

But ageing is malleable, and mounting evidence suggests that late-life ill health can be combated. In laboratory animals, including mice and rhesus monkeys, genetic, lifestyle and pharmacological interventions can increase not only the lifespan, but also the healthspan. In humans, improvements in diet and the implementation of physical exercise regimes can effect major health improvements, but better lifestyle is not enough to prevent age-related diseases.

The big hope is that 25 years from now, medical sciences will have progressed enough to enable people to have healthier and more active lives almost up until their eventual death. Going forward, the direct targeting of mechanisms of ageing, including with existing drugs, presents an opportunity to reduce disability and illness in late life. Sirolimus, an mTORC1 inhibitor, extends the lifespan of laboratory animals and in clinical trials has proved to boost the immune response of older people to vaccination against influenza. Other drugs, such as the combination of desatinib and the BCL-2 inhibitor quercetin, which kill senescent cells, are farther from the clinic but show promise. Plasma from younger mice has been shown to have a beneficial effect on the stem cell function of several tissues in older mice; work to identify the natural metabolites responsible for this effect could open up avenues for translation to the clinic. Geroprotective drugs, which target the underlying molecular mechanisms of ageing, are coming over the scientific and clinical horizons, and may help to prevent the most intractable age-related disease, dementia.

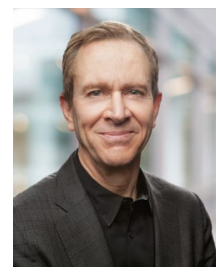

Credit: Bill \& Melinda Gates Foundation

Trevor Mundel

President of Global Health, Bill \& Melinda Gates

\section{Foundation.}

The most essential innovations in medical research over the next 25 years won't just come from the explorations of bench scientists or the emergence of new technologies. They will come from what we do-as partners across the public and private sectors-to forge a new applied research ecosystem dedicated to the rapid discovery, development and delivery of life-changing tools that have been designed with the end user in mind.

This will mean finding new ways to share clinical data that are as open as possible and as closed as necessary. It will mean moving beyond drug donations toward a new era of corporate social responsibility that encourages biotechnology and pharmaceutical companies to offer their best minds and their most promising platforms. And it will mean working with governments and multilateral organizations much earlier in the product life cycle to finance the introduction of new interventions and to ensure the sustainable development of the health systems that will deliver them. If we focus on these goals, we can deliver on the promise of global health equity.

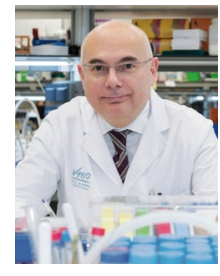

Credit: VHIO
Josep Tabernero Vall d'Hebron Institute of Oncology (VHIO); president, European Society for Medical Oncology (2018-2019).

Let's briefly skip back 25 years. In oncology, who could have predicted that the stunning advances in genome sequencing would come to shape clinical decision-making? Who could have foreseen the increasing availability of genetic patient screenings or the promise of liquid biopsy policing of disease? Very few, which is why it is a fool's errand to make sweeping predictions. But let's try.

Over the next 25 years, genomic-driven analysis will continue to broaden the impact of personalized medicine in healthcare globally. Precision medicine will continue to deliver its new paradigm in cancer care and reach more patients. Immunotherapy will deliver on its promise to dismantle cancer's armory across tumor types.

I also anticipate that AI will help guide the development of individually matched 
therapies, the harnessing and exchange of big data, and advances in telemedicine to bring crucial medical expertise to more patients everywhere. But the prospect is not all rosy. I worry about the exacerbating burden of comorbidities in cancer patients. We must collectively seek to strengthen and unify medical fields, with particular emphasis on oncology and cardiology. This is an emerging area for collaboration. Implementation research in the prevention and control of cancer will also be critical, as will be the shaping and strengthening of cancer policy-making at the global, national and regional levels.

With continued belief that scientific endeavors should be prioritized to respond to society's and citizens' needs, the scientific community must grasp future opportunities to uphold the very ethos of medicine as we continue to push boundaries in discovering new ways to extend and improve patients' lives.

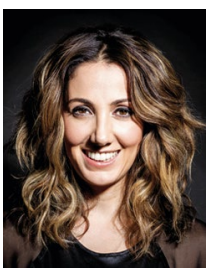

Credit: Pardis

Sabeti

\section{Pardis Sabeti}

Professor, Harvard University \& Harvard T.H. Chan School of Public Health and Broad Institute of MIT and Harvard; investigator, Howard Hughes Medical Institute.

A cataclysmic global pandemic is one of the greatest risks to humanity. Over the last 25 years, we have seen SARS, Ebola, Zika and other viruses spread undetected for months, leading to international emergencies and often devastating consequences. Even in the best US hospitals, most infectious diseases are not properly diagnosed or tracked.

But advances in two fields, genomics and information science, can transform our fight against viral threats. Ultrasensitive genome sequencing technologies are enabling the detection and characterization of viruses circulating under the radar. The advent of novel CRISPR, synthetic biology and microfluidic tools have allowed the development of rapid, ultrasensitive pointof-care diagnostics that can be deployed anywhere in the world. The resulting diagnostic and surveillance data can be integrated across healthcare nodes, from rural clinics to city hospitals, thanks to powerful new information systems. Together with advances from AI and other fields, these information systems can aid the rapid detection of infectious threats, to track their spread, and guide public health decision-making.

Over the next 25 years, the development and integration of these tools into an early-warning system embedded into healthcare systems around the world could revolutionize infectious disease detection and response. But this will only happen with a commitment from the global community.

\section{Els Torreele}

Executive director, Médecins Sans Frontières Access Campaign.

Of the many biomedical advances made by the scientific community, only those that can generate large financial profits are taken up for development by for-profit companies. This leaves many gaps-but

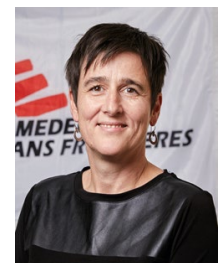
Sans Frontières
Credit: Médecins

also opportunities-in regard to developing new treatments to meet public health needs.

My hope is that the scientific community will step up and target efforts to develop innovative therapeutics and other health tools for populations across the world. This includes people affected by tuberculosis, hepatitis, Ebola, advanced HIV, neglected tropical diseases, vaccine-preventable diseases, antimicrobial resistance, snakebite-the list goes on. The creativity and brainpower of the global research community are required to find solutions addressing these grave human needs.

But to do this, we need a paradigm shift such that medicines are no longer lucrative market commodities but are global public health goods-available to all those who need them. This will require members of the scientific community to go beyond their role as researchers and actively engage in $R \& D$ policy reform mandating health research in the public interest and ensuring that the results of their work benefit many more people. The global research community can lead the way toward public-interestdriven health innovation, by undertaking collaborative open science and piloting not-for-profit R\&D strategies that positively impact people's lives globally.

Published online: 5 December 2019 https://doi.org/10.1038/s41591-019-0693-y 